\title{
Chest Wall Hemangiopericytoma-Like Solitary Fibrous Tumor of the Pleura: Case Report with Computed Tomography Findings ${ }^{*}$
}

\author{
Motohisa Kuwahara $^{1 \#}$, Hiroshi Nishimura ${ }^{1}$, Masato Iwami ${ }^{1}$, Kazuya Naritomi ${ }^{1}$, \\ Masae Mano ${ }^{1}$, Koji Inutsuka ${ }^{2}$, Keita Tokuishi' ${ }^{2}$, Akinori Iwasaki ${ }^{2}$ \\ ${ }^{1}$ Division of Surgery, Saiseikai Futukaichi Hospital, Fukuoka, Japan \\ ${ }^{2}$ Department of Chest Surgery, Fukuoka University Hospital, Fukuoka, Japan \\ Email: "KHC03152@nifty.com
}

Received October 15, 2013; revised November 15, 2013; accepted November 21, 2013

Copyright (c) 2013 Motohisa Kuwahara et al. This is an open access article distributed under the Creative Commons Attribution License, which permits unrestricted use, distribution, and reproduction in any medium, provided the original work is properly cited.

\begin{abstract}
We present a case report of a 65-year-old woman who underwent resection of a chest wall tumor. In contrast with computed tomography, the tumor exhibited hypervascularity and was fed from the 9th intercostals artery. Histologically, the tumor was diagnosed as a cellular variant of solitary fibrous tumor of the pleura, with branching "staghorn" vessels and hypervascularity. The tumor thus resembled a chest wall hemangiopericytoma.
\end{abstract}

Keywords: Solitary Fibrous Tumor; Hemangiopericytoma; Staghorn Vessels

\section{Introduction}

Solitary fibrous tumor of the pleura (SFTP) is a heterogeneous group of rare spindle-cell tumors, which includes both benign and malignant neoplasms [1,2]. Since its origin more than 60 years ago, the term hemangiopericytoma (HPC) has been used to describe a wide variety of neoplasms with certain morphological characteristics on pathologic examination: a monotonous appearance, moderate-to-high cellularity, and the presence of numerous, variably thick-walled, branching "staghorn" vessels $[3,4]$. Although fibrous to cellular SFTP, giant cell angiofibromas, and lipomatous HPCs all exhibit HPC-like characteristics, most pathologists prefer the term SFTP over HPC for these tumors, as HPC includes numerous other unrelated entities. Therefore, a high computed tomography (CT) value on contrast-enhanced CT and the existence of numerous, variably thick-walled, branching "staghorn" vessels, may be the most appropriately diagnosed as a chest wall HPC-like SFTP.

\section{Clinical Surmary}

A woman was being followed for a probable leiomyoma of the upper stomach, which was diagnosed at the age of

*Conflicts of interest: none.

${ }^{\#}$ Corresponding author.
60 based on findings at gastroscopy and on abdominal magnetic resonance imaging (MRI) studies. Five years later, when she was 65 years old, the woman was found to have left chest wall tumor on a chest MRI study (Figure 1(A)). The tumor exhibited iso-intensity to muscle on T1 images, low-intensity on T2-weighted images, and early enhancement on the dynamic study. Her chest roentgenogram at that time demonstrated a well-defined 35 $\mathrm{mm}$ tumor shadow at the left lower lung fields (Figure 1(B)). A subsequent thoracic computed tomography (CT) scan revealed a well-delineated homogenous mass of the chest wall, measuring $36 \times 34 \times 25 \mathrm{~mm}$ (Figure 2(A)). The tumor enhanced with contrast administration, suggesting that it was vascular in nature (Figure 2(B)). It had an initial CT value of 50 Hounsfield Units (HU) and enhanced CT values of $300 \mathrm{HU}$ and $150 \mathrm{HU}$ after 40 seconds and 2 minutes, respectively. A 3D-reconstruction CT study showed hypervascularization of the tumor, which was being fed from the left 9th intercostals artery (Figure 2(C)). A head MRI and an abdominal ultrasounds showed no evidence of distant metastases. Thoracoscopic resection with a utility port was performed, during which it was noted that the tumor originated from the left 9th intercostals parietal pleura (Figure 3(A)). The tumor was resected and the 9th intercostals vessels were divided by soft coagulate endo-scissors using the $\mathrm{VIO}^{\circledR}$ 


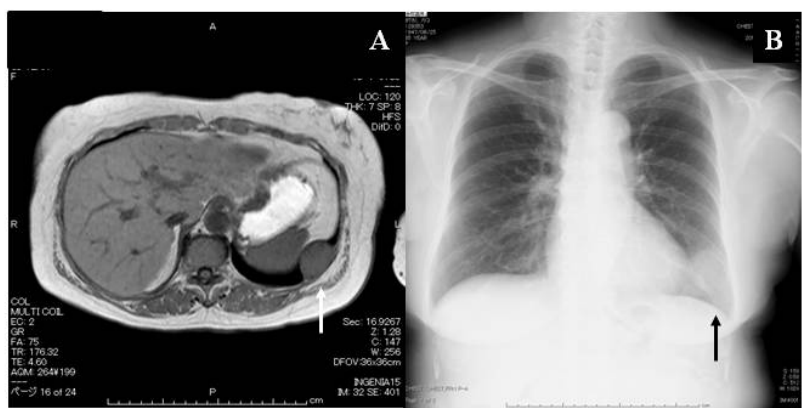

Figure 1. (A) MRI study showing the tumor of the left chest wall. (B) Chest roentgenogram demonstrating a well-defined $35 \mathrm{~mm}$ tumor shadow in the left lower lung field.

system (ERBE GmbH, Germany). No any significant bleeding occurred during the operation and the surgical borders were tumor-free. The resected specimens were divided, and on gross examination, the tumor was noted to be whitish and dense (Figure 3(B)). Microscopic evaluation revealed a solid mesenchymal mass composed of indistinct fusiform to spindle-shaped and rounded cells arranged around slit-like and branching thin-walled "staghorn” vessels (Figure 3(C)). No pleomorphism or atypia was detected and the mitotic count was extremely low. On immunohistochemical staining, the tumor cells were positive for CD34 (Figure 3(D)) and negative for AE1/AE3 and S-100. These findings led to the pathologic diagnosis of a cellular variant of SFTP. The woman's postoperative course was uneventful and she was discharged to home after 18 days. She was subsequently followed in our out-patient clinic.

\section{Disscusion}

SFTP have been referred to by a variety of names that reflect their clinical course and controversies surrounding their histogenesis (e.g., benign mesotheliomas, localized fibrous mesotheliomas or subpleural fibromas) [1]. Typically, SFTP are solitary and arise from the visceral pleura. Almost 50\% are pedunculated, with the vascular supply contained within their pedicles. The treatment of choice for SFTP is complete surgical excision. Thoracoscopic approaches can be safely used to excise small pedunculated tumors.

HPC arise from an abnormal proliferation of pericytes, which are contractile cells that usually lie outside the endothelial cell of capillary vessels [3,4]. These tumors most common occur in the muscles of the lower extremities and retroperitoneum, but they are occasionally seen in the thoracic cavity. SFTP are a type of HPC-like neoplasm. There is no proof to date suggesting the relationship of these tumor pericytes. Radiographic findings are nonspecific as well: HPCs appear as well-circumscribed, radiopaque masses on chest X-ray. CT scan findings reveal vascularized soft tissue masses with a density re- sembling ground glass, which often displace neighboring structures.

CT scans of small SFTP typically demonstrate homogeneous, well-defined, non-invasive, lobular, soft tissue masses, which typically abut a pleural surface [2]. They may form obtuse angles against the adjacent pleural surface. Calcification may be observed in some SFTP, with either benign or malignant histological features. The presence of calcifications has been documented in 7\% $26 \%$ of SFTPs, primarily in areas of necrosis within large tumors. Local invasion is rare, and lymphadenopathy is not a feature of SFTP. Chest wall involvement has been observed by CT in $8 \%$ of cases, and is manifested by sclerosis or pressure erosion of the adjacent ribs. This pressure erosion is a characteristic feature of chest wall and mediastinal neoplasms of neurogenic origin and is rarely reported in association SFTP.

SFTP typically exhibit intermediate-to-high attenuation on enhanced CT imaging because of high density of collagen and the abundant capillary network within these tumors. SFTP often exhibit greater attenuation that other soft tissues in the thorax and enhancement may correlate with the vascular nature of these tumors. In Chu et al's study of 16 SFTP that were valuated by both plain and enhanced CT imaging, all tumors demonstrated significant enhancement [5]. The mean plain $\mathrm{CT}$ and contrast enhanced CT values were $37 \mathrm{HU}$ and $60 \mathrm{HU}$, respectively. In our case, the enhanced CT value was $300 \mathrm{HU}$, which was consistent with hypervascularity and thus supported the diagnosis of an HPC-like tumor. However, contrast material enhancement may not occur with all SFTP.

In SFTP, enhancement on contrast CT is typically heterogeneous with central areas of low attenuation that, correlate with myxoid change, hemorrhage, necrosis, or cystic degeneration. This heterogeneity may difficult to see prior to intravenous administration of contrast material. In general only benign SFTPs exhibit homogeneous attenuation, which is more commonly seen on unenhanced scans. Homogeneity of attenuation may indirectly relate to the size of the tumor, as small tumors are less likely to exhibit necrosis. Hemorrhage, necrosis, or cystic change was absent in $88 \%$, with homogenous attenuation on unenhanced scans and in all SFTPs with homogenous attenuation on contrast-enhanced CT scans.

Angiography is an important diagnostic tool for SFTP as it can ascertain the vascular supply of these tumors, which typically enters through their pedicles. Demonstration of the blood supply arising from the inferior phrenic, intercostals, or internal mammary arteries may be a helpful clue to the extrapulmonary origin of SFTP. Furthermore, preoperative angiography with arterial embolization may be useful strategy reduce intraoperative bleeding. Our 3D-reconstruction CT images were simi- 


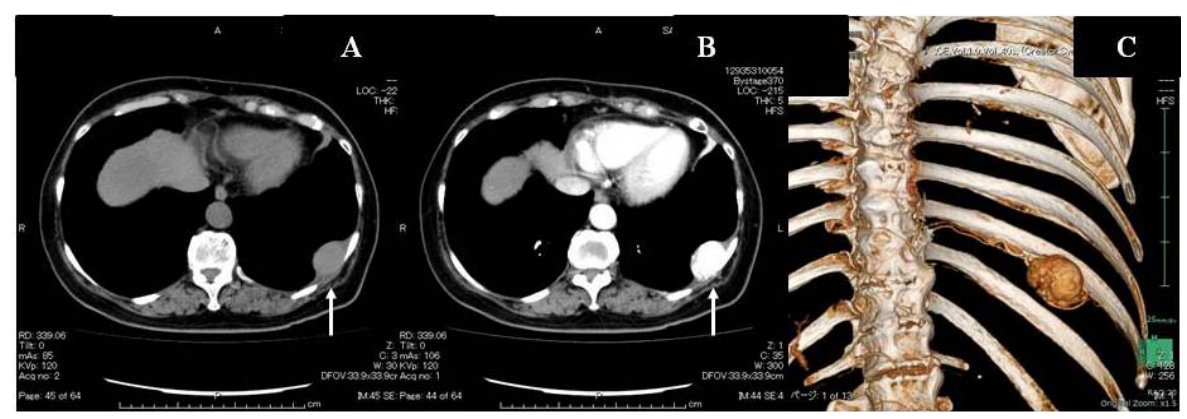

Figure 2. (A) CT scan of the thorax showing a well-delineated homogenous, $36 \mathrm{~mm}$ mass of the left chest wall. (B) Enhanced CT imaging at 40 seconds showing the tumor. The enhanced CT value at that time was $300 \mathrm{HU}$. (C) 3D-reconstruction study showing the tumor's hypervascularization and arterial inflow from the left 9th intercostals artery.

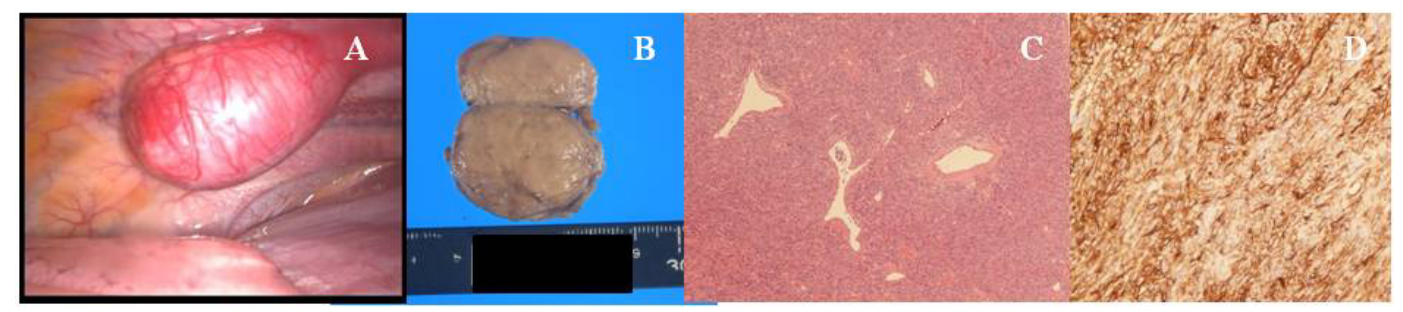

Figure 3. (A) Intraoperative view showing the tumor originating from the left 9th intercostals parietal pleura. (B) Gross examination sowing a whitish and dense tumor. (C) Histological examination showing a solid mesenchymal mass composed of indistinct fusiform to spindle-shaped and rounded cells arranged around slit-like and branching thin-walled "staghorn" vessels (hematoxylin and eosin staining). (D) Immunohistochemically staining showing that the tumor cells stained positive for CD34.

larly useful in demonstrating the tumor's blood supply from the 9th intercostals artery as affirming that there was good instrument control for hemorrhage embolization. Histopathologic examination of HPCs reveals tumors with cellar and vascular components: dense spindle-shaped and polymorphic tumor cells, typical staghorn-structured dilated vascular endothelial cell vessels, and distribution of tumor cells along vessels. Therefore, an HPC-like growth pattern was confirmed in our case; however, because of the lack of clear classification criteria and the major overlap between SFTP and other benign or malignant lesions (such as HPCs) that share histologic elements, we encountered difficulty with classifying this case into a known entity. In accordance with most recent classification recommendation, we assigned it to the SFTP group, as a predominantly cellular variant of SFTP.

\section{Conclusion}

In summary, we described a case of a chest wall SFTP, which was surgically resected. The tumor exhibits a high enhanced-CT value, reflecting its hypervascularity. IT was composed of indistinct fusiform to spindle-shaped and rounded cells arranged around slit-like and branching thin-walled "staghorn" vessels, similar to those seen in HPCs.

\section{REFERENCES}

[1] W. Abu Arab, "Solitary Fibrous Tumours of the Pleura," European Journal Cardio-Thoracic Surgery, Vol. 41, No. 3, 2012, pp. 587-597. http://dx.doi.org/10.1093/ejcts/ezr009

[2] Y. Zhu, K. Du, X. Ye, D. Song and D. Long, "Solitary Fibrous Tumors of Pleura and Lung: Report of Twelve Cases," Journal of Thoracic Disease, Vol. 5, No. 3, 2013, pp. 310-313.

[3] P. Bicakcioglu, E. Aydin, A. Celik, F. Demirag and N. Karaoglanoglu, "Primary Classical Hemangiopericytomas of Thorax,” The Annals of Thoracic Surgery, Vol. 94, No. 1, 2012, pp. 255-259. http://dx.doi.org/10.1016/j.athoracsur.2012.03.063

[4] N. Penel, E. Y. Amela, G. Decanter, Y. M. Robin and P. Marec-Berard, "Solitary Fibrous Tumors and So-Called Hemangiopericytoma,” Sarcoma, Vol. 2012, 2012, Article ID: 690251. http://dx.doi.org/10.1155/2012/690251

[5] X. Chu, L. Zhang, Z. Xue, et al., "Solitary Fibrous Tumor of the Pleura: An Analysis of Forty Patients," Journal of Thoracic Diseases, Vol. 4, No. 2, 2012, pp. 146-154. 